\title{
Reduced Order Chemical Kinetic Modeling for a Hydrogen Fueled Radical Farming Scramjet
}

\author{
Raoul Mazumdar ${ }^{1, *}$, Hideaki Ogawa ${ }^{1,2}$, Adrian Pudsey 1 \\ ${ }^{1}$ School of Engineering, RMIT University \\ Victoria, Australia \\ Raoul.mazumdar@student.rmit.edu.au; Adrian.pudsey@rmit.edu.au \\ ${ }^{2}$ Department of Aeronautics and Astronautics, Kyushu University \\ Fukuoka, Japan \\ hideaki.ogawa@aero.kyushu-u.ac.jp
}

\begin{abstract}
Computational modeling for scramjets and other high-speed reacting fluids often requires significant computing resources. The need to capture complex flow phenomena at high-speeds requires the use of large grids. This numerical demand is further compounded by the chemistry, which must be resolved across the entire domain. This work investigates the reduction of a detailed hydrogen mechanism, containing some 33 reactions and 10 species. The effectiveness of the mechanism reduction is examined by applying the results to a two-dimensional radical farming scramjet geometry. The results show that a 5-step reduced hydrogen mechanism with chemical kinetic rates optimized using laminar burning velocities can potentially replicate aspects of a detailed hydrogen mechanism. On current computing infrastructure, a speed up to 2.7 times has been achieved with the reduced models in comparison to the detailed model in the hydrogen combustion in a two-dimensional domain.
\end{abstract}

Keywords: Reduced-order, Hydrogen, Radical farming, Scramjet, Chemical kinetics

\section{Introduction}

Chemical mechanisms are a primary ingredient for any chemically reacting flow simulation, whether they be for the modeling of hypersonic propulsion, automotive engines, or even refinery exhaust stacks. These mechanisms describe the chemistry generally through finite elementary reactions, and are fed into the various computational fluid dynamics software packages. For small fuels such as hydrogen, the chemical mechanisms may have only a few dozen reactions or species. For complex geometries and high speed flows where fine mesh sizes are warranted, the numerical requirement to simulate the system for even a hydrogen mechanism increases substantially. Keeping this in consideration, if the detailed chemistry can be replicated by a smaller mechanism, using only the critical reactions and species necessary, the computational resources required can be reduced. This provides an engineer or scientist with the ability to model their combustion or reacting simulation at a fraction of the time with a reduced chemical mechanism.

Hydrogen fuel is well used in the hypersonic air-breathing propulsion community, having been employed in numerous computational and experimental studies over the past few decades [1-5]. The ease at which the fuel is ignited in supersonic airstreams and its high heat release makes hydrogen of particular use for hypersonic applications. Such properties have also made it a subject of investigation in other areas of engineering. As such, the research field has produced a myriad of chemical kinetic mechanisms for hydrogen combustion, including a number of reduced mechanisms [6-8]. Ströhle and Tore [8] developed multiple hydrogen mechanisms for the application to gas turbine simulations, using a variety of one-dimensional and zero-dimensional models to guide the reduction procedure. Bovin [10] conducted extensive research for reduced hydrogen mechanisms, creating several models including a 12 reaction and 9 species mechanism, and a 3 reaction mechanism with quasi-steady approximations for steady-state species. The primary difficulties of the current reduced hydrogen mechanisms in circulation are that they are not optimized for the conditions experienced by hypersonic air-breathing applications, and they use chemical rate expressions that are sometimes difficult to implement in generic computational fluid dynamics software packages. 
The current work is conducted to address critical questions in the realm of reduced chemical mechanisms for hypersonic air-breathing applications, e.g., can a reduced hydrogen mechanism be created that mimics a detailed chemical kinetic mechanism? If so, what are the key zero and one-dimensional targets used and are they effective for this specific application area? To achieve this objective, a detailed hydrogen mechanism is taken from Jackimowski [5], for which the reduced mechanism attempts to replicate the detailed hydrogen mechanism. In order to study the effects of the detailed and reduced mechanisms on a realistic hypersonic geometry, the mechanisms are simulated in a twodimensional premixed radical farming scramjet. This particular simulation is based on the work by McGuire et al. [11], in which a scramjet was studied using a simplified premixed inflow condition, with ignition enhancement using a shocktrain to accelerate fuel decomposition. The generation of a reduced chemical mechanism in this work, in addition to a detailed reduction approach, lowers the cost of adoption for both engineers and scientists to apply either these methods to other fuels, or adopt the reduced mechanism for their own simulations in hypersonics.

\section{Numerical Approach}

\subsection{Reduced Mechanism Approach}

The approach taken in this work is to extract key chemical processes in hydrogen chemistry and employ them as a reduced mechanism. Critical reactions are thus taken from the publication of Browne and Shepard [12], forming a reduced 5-step mechanism. Equation (1) represents a radical branching reaction and Eq. (2) is a radical propagation reaction. Equation (3) represents an important initiation reaction, consuming oxygen and hydrogen to form two radical species. Finally, the last two reactions of Eqs. (4)-(5) in the 5-step method, are radical chain propagation and branching, respectively. By using this reduced chemical mechanism, the bulk of the chemical reactions are rejected unlike in a detailed chemical mechanism. The premise for this exclusion is that the core reactions are modeled in the 5-step mechanism, and thus the subsequent error between the 5-step and detailed mechanism is small. In order to further reduce this error, the chemical kinetic rates can be optimized in order to replicate the laminar burning velocity of the detailed mechanism. The laminar burning velocity is an ideal method to base the rate optimization and mechanism performance, as the parameter has been employed for mechanism validation in other reduction studies [9].

$$
\begin{aligned}
\mathrm{H}+\mathrm{O}_{2} & \leftrightarrow \mathrm{OH}+\mathrm{O} \\
\mathrm{H}+\mathrm{O}_{2}+\mathrm{M} & \leftrightarrow \mathrm{HO}_{2}+\mathrm{M} \\
\mathrm{H}_{2}+\mathrm{O}_{2} & \leftrightarrow \mathrm{HO}_{2}+\mathrm{H} \\
\mathrm{OH}+\mathrm{H}_{2} & \leftrightarrow \mathrm{H}_{2} \mathrm{O}+\mathrm{H} \\
\mathrm{O}+\mathrm{H}_{2} & \leftrightarrow \mathrm{OH}+\mathrm{H}
\end{aligned}
$$

Laminar burning velocities are critical to any application involving reacting flows, as they represent a fundamental property of a mixture and its burning characteristics. In order to model the laminar flame speed for the detailed and reduced mechanisms, this work utilizes an open-source chemical kinetic software package; Cantera [13]. The package also contains a suite of one-dimensional tools including models for laminar flame speeds, which solves the governing equations for mass, continuity, energy, and species as shown in Eqs. (6)-(9). As discussed within Cantera's manual, the parameter $V$ represents radial velocity, $\rho$ the density, $u$ the axial velocity, and $z$ the centerline of flame propagation. $\Lambda$ represents the pressure eigenvalue, and $\mu$ is dynamic viscosity. $\lambda$ is the thermal conductivity, $j_{k}$ is diffusive mass flux of species $k$, and $c_{p, k}$ is the heat capacity at constant pressure of species $k$. Enthalpy, mass fraction, molecular weight, and molar species production of species $k$ are represented by $h_{k}, Y_{k}, W_{k}$ and $\dot{\omega}_{k}$, respectively. By solving the equations and applying the right boundary conditions for the case at hand, a variety of different one-dimensional flows can be modeled, as discussed in Kee et al. [14].

Continuity

$$
\frac{\delta \rho u}{\delta z}+2 \rho V=0
$$


Radial momentum

$$
\rho u \frac{\delta V}{\delta z}+\rho V^{2}=-\Lambda+\frac{\delta}{\delta z}\left(\mu \frac{\delta V}{\delta z}\right)
$$

Energy

$$
\rho C_{p} u \frac{\delta T}{\delta z}=\frac{\delta}{\delta z}\left(\lambda \frac{\delta T}{\delta z}\right)-\sum_{k} j_{k} c_{p, k} \frac{\delta T}{\delta z}-\sum_{k} h_{k} W_{k} \dot{\omega}_{k}
$$

Species

$$
\rho u \frac{\delta Y_{k}}{\delta z}=-\frac{\delta j_{k}}{\delta z}+W_{k} \dot{\omega}_{k}
$$

The laminar flame is modeled as a freely propagating adiabatic premixed hydrogen flame and is computed for a range of equivalence ratios from 0.5 to 3 . The flame is calculated for both the reduced and detailed mechanisms at a pressure of $1 \mathrm{~atm}$, and at a temperature of $300 \mathrm{~K}$. In order to minimize the difference between the reduced mechanism's laminar burning velocities, the reaction rates are multiplied by a scalar operation until the error becomes minimum as defined in Eq. (10). The process is depicted in Fig. 1, where successive iterations are performed using the Nelder-Mead minimization routine from Scipy [15].

$$
\text { Error }=\sum_{i}^{n}\left|S_{l, \text { detailed }}-S_{l, \text { reduced }}\right|
$$

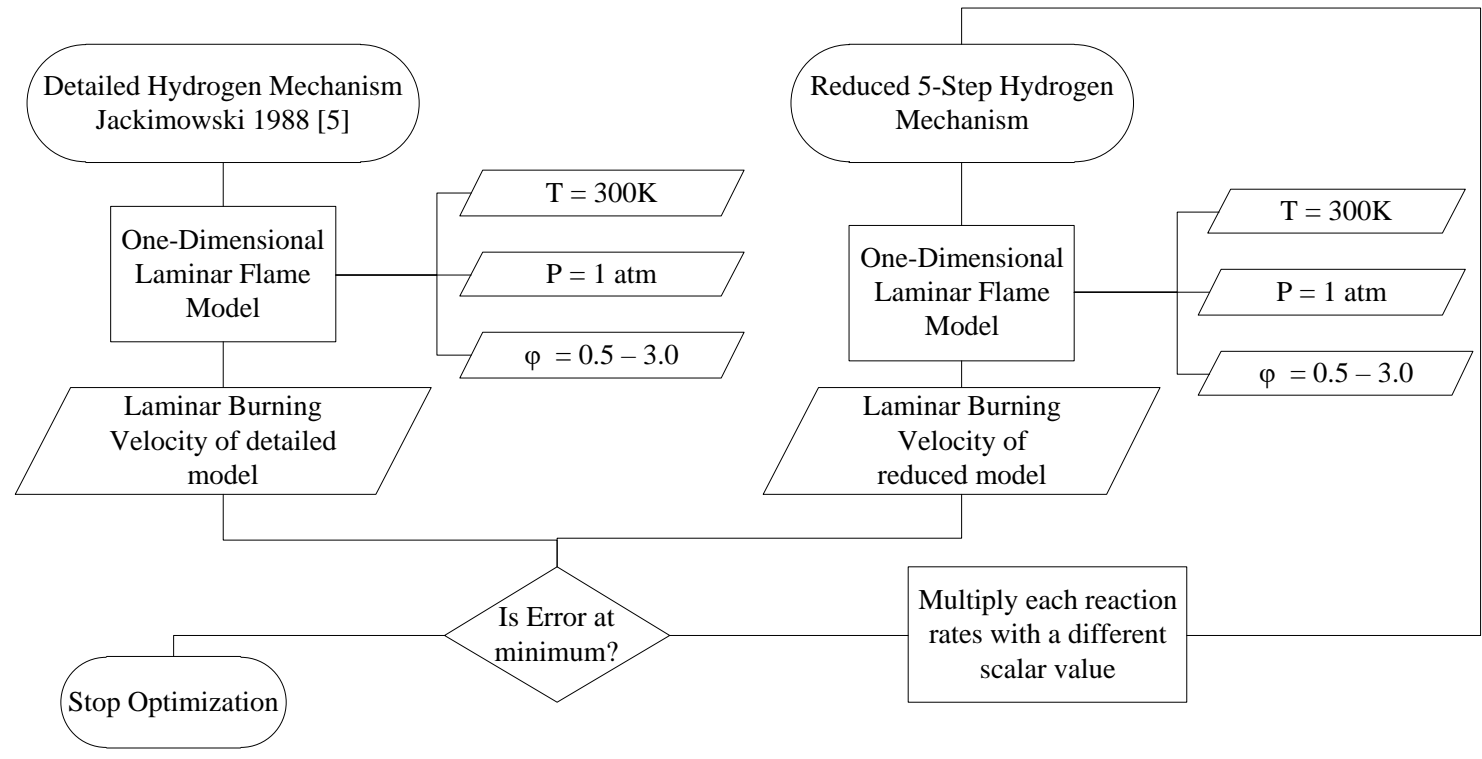

Fig. 1: Flow chart describing the optimization procedure for tuning the 5-step reduced mechanism to replicate the laminar burning velocity of the detailed mechanism.

The outcome of the optimization is presented in Fig. 2, where the laminar burning velocity for the detailed mechanism, 5-step mechanism, and the optimized 5-step mechanisms are displayed. The dashed line represents the model without optimization, using chemical kinetic rates that are sourced from the literature [5,12]. The smaller-dotted line represents the optimized solution, where chemical kinetic rates are multiplied by a scalar that minimizes the error function. The resultant mechanism closely matches the detailed mechanism for the laminar burning velocities, although there remain small errors for the smaller equivalence ratios sampled at low values. Now that the mechanism has been reduced, the next step is to examine the validity of the mechanism for a hypersonic relevant 


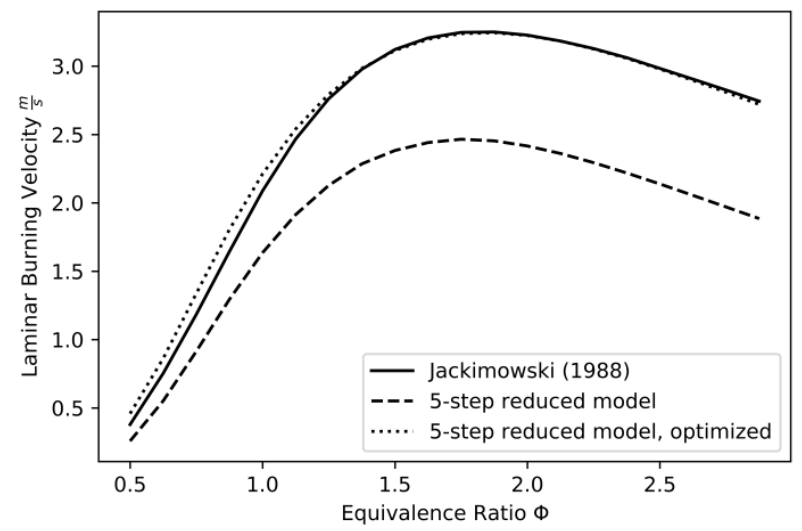

Fig. 2: Laminar flame speed plot at 1 atm and 300K. Full detailed hydrogen model using Jackimowski (1988) [5]. 5-step reduced model, using unoptimized rates. 5-step model optimized, includes kinetic rates changed in order to replicate the detailed hydrogen

Jackimowski (1988) [5] model at these conditions.

\subsection{Flow Solver}

The computational fluid dynamics (CFD) software package CFD++ [16] by Metacomp is utilized to perform reacting simulations in a two-dimensional domain. CFD++ is capable of solving a variety of flows including inviscid/viscous, incompressible/compressible, and steady/unsteady flows. Given the geometry considered in this work, recirculating flow behavior is considered to be negligible. Compressible Reynolds Averaged Navier Stokes (RANS) equations are solved for the flow domain. The flowfield is computed up to the wall without wall functions. Turbulence is modeled using the shear-stress transport model, which is well used in a variety of industrial applications [17]. These solver parameters are employed to perform reacting flow simulations with both the detailed and reduced hydrogen mechanisms.

\subsection{Domain and Mesh Sensitivity}

The geometry is based on the work of Mcguire et al. [11], who investigated a range of input conditions in order to study radical farming behavior in two-dimensional scramjet ducts. Similarly, the same methodology and geometry are adopted from the paper (Fig. 3). The modeled domain is downstream of a 9-degree ramp and consists of a duct with a 3 -degree intake shroud. The main body of the duct is $10 \mathrm{~mm}$ in height, and $298.61 \mathrm{~mm}$ long.

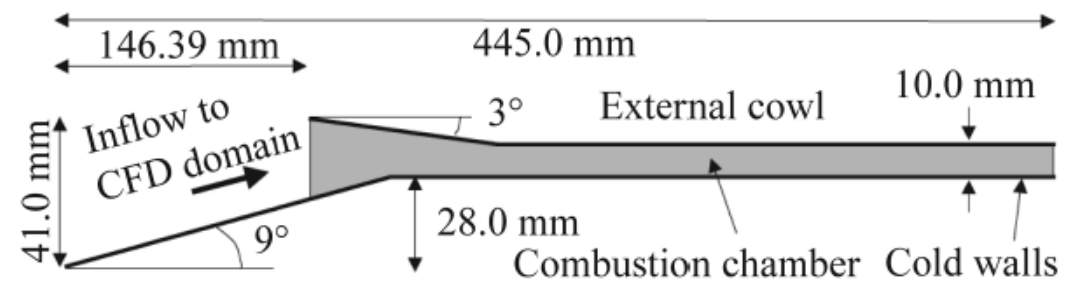

Fig. 3: CFD domain sourced from McGuire et al. [11], and employed in this study.

A mesh sensitivity study was conducted to identify the point at which fluid results were independent of the grid resolution. The approach used the Richardson extrapolation [18] to extrapolate the values of pressure, temperature and species fraction near the wall. The fine mesh comprising 190,000 cells was found to produce values closely matching the Richardson values. This indicates that the fine mesh is adequate for simulating the proceeding combustion simulations and is used henceforth. The input flow conditions for this domain consist of a hydrogen-air mixture at an enthalpy of $4.90 \mathrm{MJ} / \mathrm{Kg}$, a static pressure of $11900 \mathrm{~Pa}$, a static temperature of $300 \mathrm{~K}$, and a total velocity of $2835 \mathrm{~m} / \mathrm{s}$. 
The walls are modeled as isothermal at $293 \mathrm{~K}$, assuming the condition of an experimental apparatus. These flow conditions correspond to a radical farming flow regime, and for a flight vehicle at Mach 8.

\section{Result}

Figure 4 presents three flowfields: non-reacting flow, reacting flow with the detailed hydrogen mechanism, and reacting flow with the reduced hydrogen mechanism. The flowfield shows a series of incident and reflected shock-waves, and expansion waves propagating through the duct, producing pockets of high temperature and pressure. The differences between the detailed and reduced hydrogen mechanism are minimal near the inlet, since the flow is still too cold to undergo significant chemical reactions. As the flow proceeds down the duct, the fuel begins to decompose in the pockets behind the reflected shocks, leading to increases in pressure and temperature. Deviations between the reduced and detailed models are even more pronounced near the outlet, where the detailed model reaches higher pressure and temperatures than the reduced model. In order to gain insight and compare the reacting flows for the detailed and reduced mechanisms, a streamline is placed near the lower wall of the domain, as observed in Fig. 4. Subsequent analysis examines the flow properties and chemistry at points along the domain on the streamlines displayed in Fig. 4 for the non-reacting, detailed and reduced models.

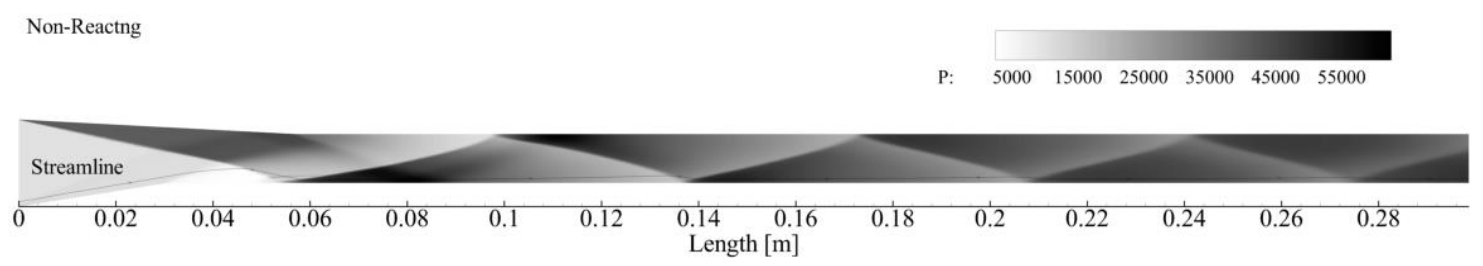

(a) Non-reacting flowfield

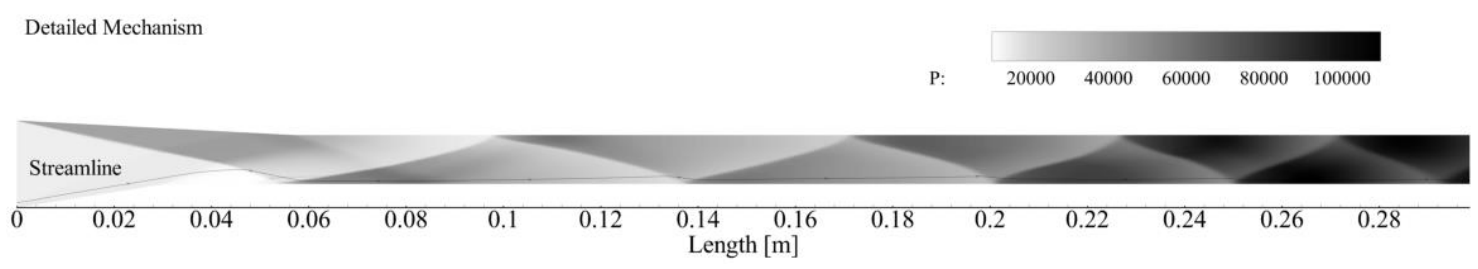

(b) Reacting flowfield with detailed hydrogen mechanism [5]

Reduced Mechanism

P: $\quad 10000 \quad 20000 \quad 30000 \quad 40000 \quad 50000 \quad 60000 \quad 70000$

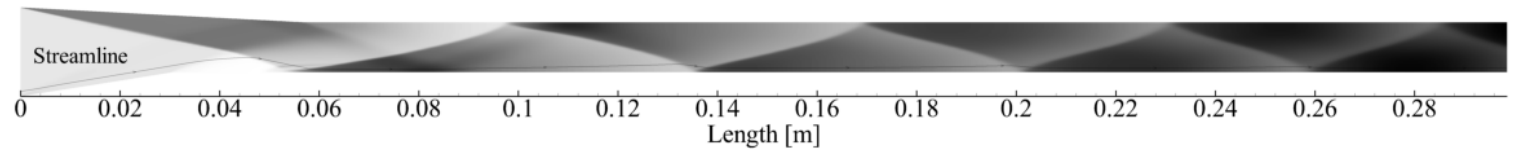

(c) Reacting flowfield with reduced hydrogen mechanism

Fig. 4: Pressure contours of non-reacting (a), detailed hydrogen (b) and reduced 5-step hydrogen mechanism (c).

The flow properties along the streamline are plotted, including species concentrations, static temperatures, pressures, and Mach number. In Fig. 5(a), it is evident that along the wall the pressure and temperature are significantly higher in the detailed model than the reduced model; these results are likely to be attributed to incomplete combustion in the reduced model. This is supported in Fig. 5(b), which indicates that $\mathrm{H}_{2} \mathrm{O}$ species mass fractions are lower in the reduced model as compared to the detailed model. The $\mathrm{H}_{2}$ species in the reduced model is also significantly higher than the detailed model, further underpinning that incomplete consumption of the fuel has resulted in the lower levels of temperature and pressure. 


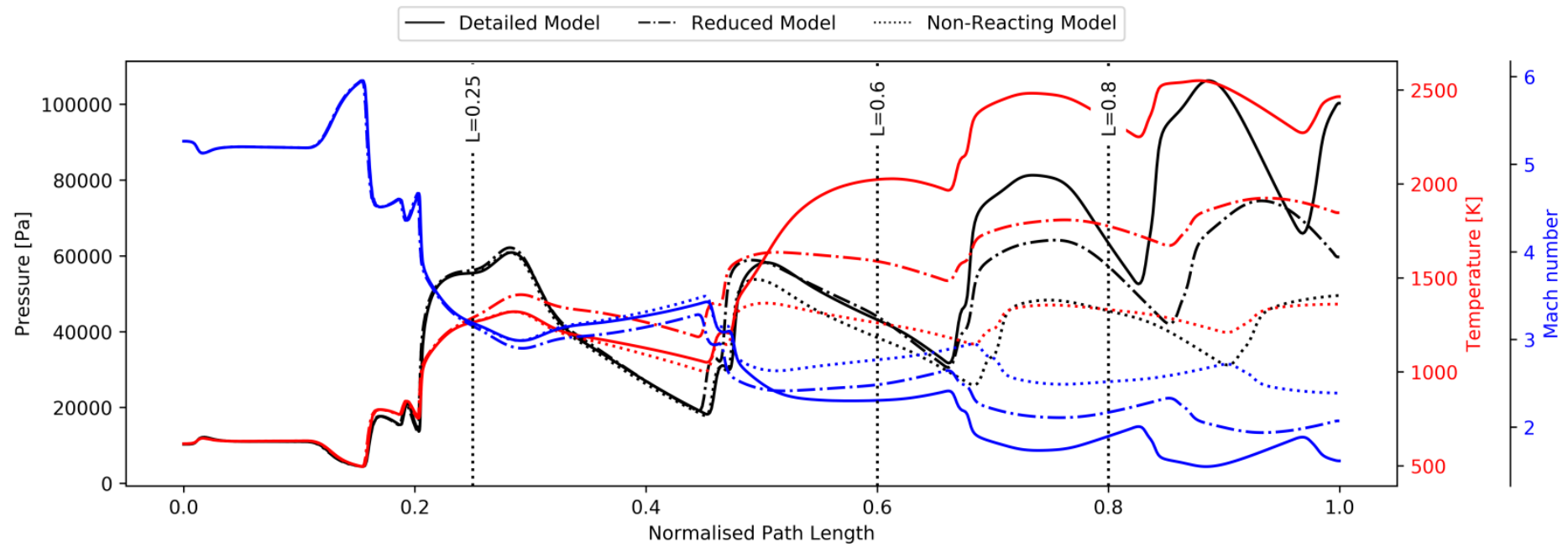

(a) Distributions of static pressure, temperature and Mach number along the streamline.

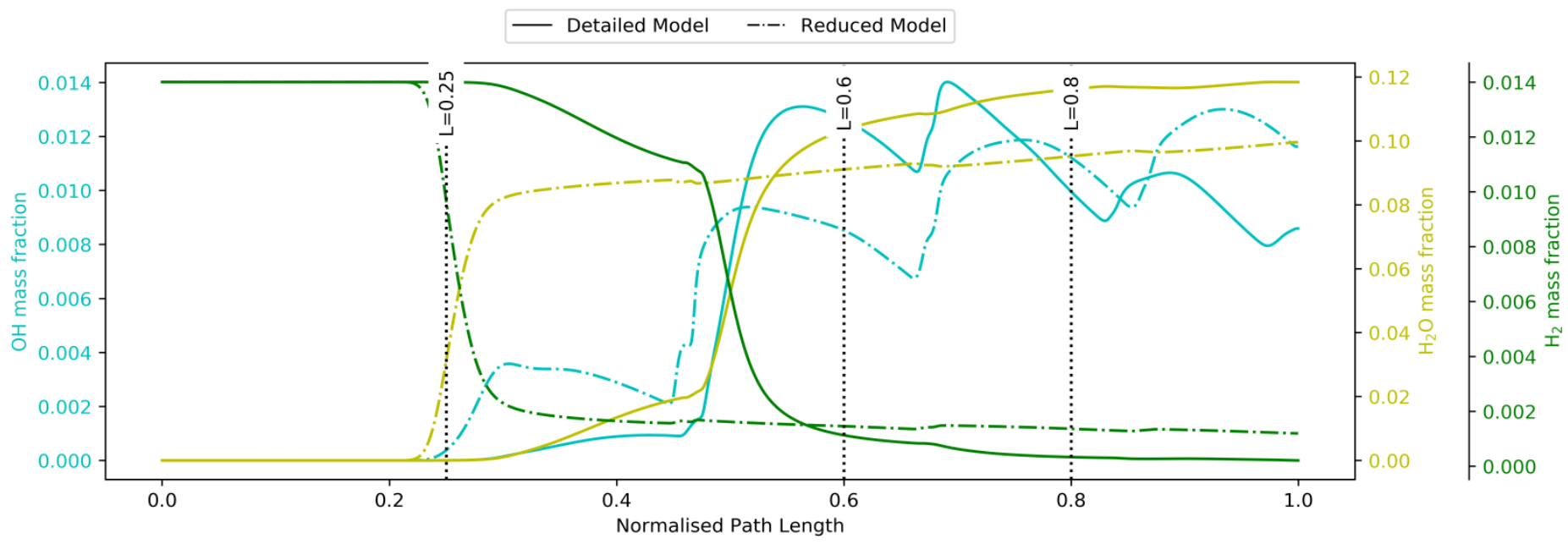

(b) Distributions of chemical species mass concentration for $\mathrm{OH}, \mathrm{H}_{2} \mathrm{O}$ and $\mathrm{H}_{2}$ along the streamline.

Fig. 5: Plot of macroscopic properties along the streamline including chemical species mass fractions for the detailed, reduced, and non-reacting models.

To explain the cause for these discrepancies between the chemical models it is necessary to probe into the chemistry and pathway dominance along the streamline. Fig. 6 depicts the H-atom transfer, highlighting where the dominant migration of $\mathrm{H}$-atoms is within that system in those specific species concentrations, temperatures, and pressures. The detailed model shows that in the initial hot pocket at a normalized distance of 0.25 along the domain length and stream, the fuel $\mathrm{H}_{2}$ is breaking down rapidly to form $\mathrm{OH}$ and $\mathrm{H}$ radicals. As the flow reaches the next hot pocket of gas at a normalized distance of 0.6 , the radical pool is rapidly consuming the primary fuel species $\mathrm{H}_{2}$ and leading to oxidation into $\mathrm{H}_{2} \mathrm{O}$. By the time the mixture has reached the normalized length of 0.8 along the streamline, much of the fuel has been consumed and the dominant pathway is the consumption of $\mathrm{OH}$ radicals to form $\mathrm{H}_{2} \mathrm{O}$. In comparison to this behavior, the reduced model shows limited deviation in its chemical characteristics across the domain, and more importantly, the model's end products are $\mathrm{H}_{2} \mathrm{O}$ and $\mathrm{HO}_{2}$, which is not realistic since $\mathrm{HO}_{2}$ itself is a radical. The oxidation pathway to $\mathrm{H}_{2} \mathrm{O}$ is highly exothermic, and thus not producing sufficient amounts of $\mathrm{H}_{2} \mathrm{O}$. The simulation is, therefore, indicative of lower pressure and temperature for the case of the reduced model. 


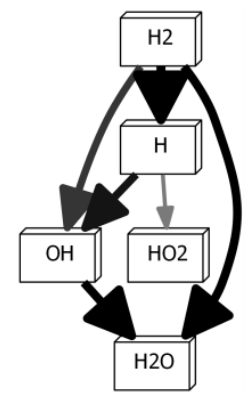

(a) Detailed Model at normalized length of 0.25

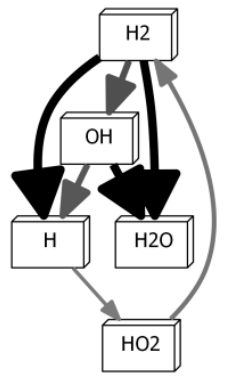

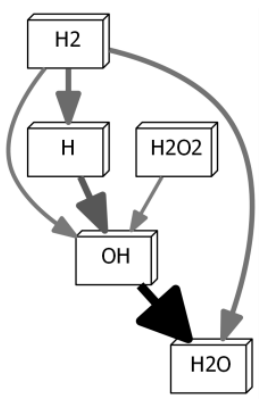

(b) Detailed Model at normalized length 0.6

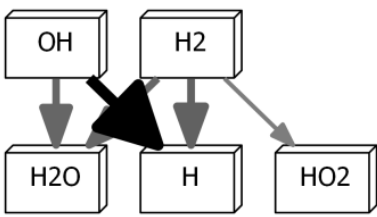

(e) Reduced Model at normalized length of 0.6

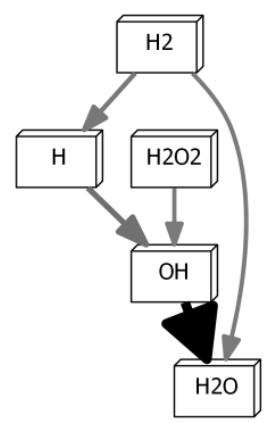

(c) Detailed Model at normalized length 0.8

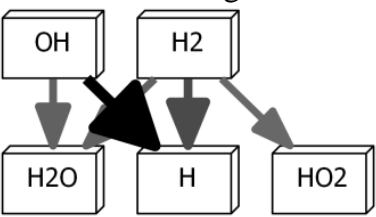

(f) Reduced Model at normalized length of 0.8

(d) Reduced Model at normalized length of 0.25

Fig. 6: H-atom pathway plots depicting the direction and prevalence of $\mathrm{H}$-atoms transfers between species. Black thick lines indicate primary dominance, grey lines represent secondary significance, thin lines denote lower preference. Pathways are depicted along the streamline of the domain at the normalized lengths of $0.25,0.6$ and 0.8 for the detailed and reduced models.

\section{Conclusion}

The present study has investigated the process of creating a reduced chemical kinetic mechanism for application in hypersonic air-breathing propulsion. In the process, it has been shown that using a sub-mechanism of published works as a reduced chemical mechanism can be effective, especially in that the mechanism's laminar burning velocity is similar to that of the detailed mechanism. While the smaller mechanism is an adequate first attempt, particular care must be taken so that $\mathrm{H}_{2} \mathrm{O}$ is the dominant end product of the pathway analysis. It is clear that careful selection of appropriate elementary reactions, comparison of the chemical pathways, and laminar burning velocity are vital for ensuring the creation of suitable reduced mechanisms.

Using laminar burning velocity alone is not sufficient to ensure the generation of an adequate reduced mechanism. However, in this case, we were able to reproduce the properties of the detailed mechanism fairly well with a reduced mechanism that lacked the correct chemical pathways. Refining this process is of considerable importance in creating a robust approach to chemical mechanism reduction for hydrogen and other fuels. Decreasing the numerical demand in CFD has long been a primary interest and requirement for many engineers and scientists, and until these questions can be addressed it is unlikely to be acomplished.

\section{Acknowledgments}

The CRC Programme supports industry-led collaborations between industry, researchers and the community. The authors would like to thank the support of the CRC-P, RMIT University, and the high-performance computing resources and support from the National Computational Infrastructure (NCI), as well as the Australian government's higher education training scheme. 


\section{Reference}

[1] Oevermann, M. "Numerical investigation of turbulent hydrogen combustion in a SCRAMJET using flamelet modeling." Aerospace science and technology 4, no. 7 (2000): pp. 463-480.

[2] Gerlinger, P., Peter S., Markus K., Fernando Schneider, and Manfred Aigner. "Numerical investigation of mixing and combustion enhancement in supersonic combustors by strut induced streamwise vorticity." Aerospace Science and Technology 12, no. 2 (2008): pp. 159-168.

[3] Micka, D. J., and James F. Driscoll. "Combustion characteristics of a dual-mode scramjet combustor with cavity flameholder." Proceedings of the combustion institute 32, no. 2 (2009): pp. 2397-2404.

[4] Billig, F. S. "Research on supersonic combustion." Journal of Propulsion and Power 9, no. 4 (1993): pp. $499-514$.

[5] Jachimowski, C. J. "An analytical study of the hydrogen-air reaction mechanism with application to scramjet combustion." NASA TN-D-5315 (1988).

[6] Massias, A., Diamantis, D., Mastorakos, E., and Goussis, D. A. "Global reduced mechanisms for methane and hydrogen combustion with nitric oxide formation constructed with CSP data." Combustion theory and modelling 3, no. 2 (1999): pp. 233-257.

[7] Williams, F. A. "Detailed and reduced chemistry for hydrogen autoignition." Journal of Loss prevention in the Process Industries 21, no. 2 (2008): pp. 131-135.

[8] Ju, Y., and Niioka, T. "Reduced kinetic mechanism of ignition for nonpremixed hydrogen/air in a supersonic mixing layer." Combustion and flame 99, no. 2 (1994): pp. 240-246.

[9] Ströhle, J., and Tore M. "Reduction of a detailed reaction mechanism for hydrogen combustion under gas turbine conditions." Combustion and flame 144, no. 3 (2006): pp. 545-557.

[10] Boivin, P. "Reduced-kinetic mechanisms for hydrogen and syngas combustion including autoignition." (2011).

[11] McGuire, J. R., R. R. Boyce, and Mudford, N. R. "Radical-farm ignition processes in two-dimensional supersonic combustion." Journal of Propulsion and Power 24, no. 6 (2008): pp. 1248-1257.

[12] Browne, S., Liang, Z., and Shepherd, J. E. "Detailed and simplified chemical reaction mechanisms for detonation simulation." Fall 2005 Western States Section of the Combustion Institute (2005).

[13] Goodwin, D. G., Harry K. M., and Raymond L. S. "Cantera: An object-oriented software toolkit for chemical kinetics, thermodynamics, and transport processes. Version 2.2. 1." Cantera Developers, Warrenville, IL (2016).

[14] Kee, R. J., Coltrin, M. E., and Glarborg, P.. Chemically reacting flow: theory and practice. John Wiley \& Sons, 2005.

[15] Jones, E., Oliphant, T., \& Peterson, P. "others. SciPy: Open source scientific tools for Python." Web http://www. scipy. org (2001).

[16] Chakravarthy, S., Peroomian, O., Goldberg, U., \& Palaniswamy, S. "The CFD++ computational fluid dynamics software suite." In AIAA and SAE, 1998 World Aviation Conference, p. 5564. 1998.

[17] Menter, F. R. "Review of the shear-stress transport turbulence model experience from an industrial perspective." International journal of computational fluid dynamics 23, no. 4 (2009): pp. 305-316.

[18] Phillips, T. S., \& Roy, C. J. "Richardson extrapolation-based discretization uncertainty estimation for computational fluid dynamics." Journal of Fluids Engineering 136, no. 12 (2014): pp. 121401. 